\title{
EDITORIAL
}

\section{MICROLITHOGRAPHY AND AVIATION}

Microlithography is a fast moving technology. Since Kilby invented the integrated circuit in 1958, microlithography has come a long way. The minimum feature has reduced from hundreds of micrometers to hundredths of micrometers, i.e., by at least 4 orders of magnitude. Aviation started in 1903 when the Wright Brothers successfully made a powered flight of a heavier-than-air aircraft. The range of flight started from $120 \mathrm{ft}$ in the first Wright Brothers' aircraft. It is now 8000 nautical miles on a Boeing 747-8, an increase of more than 5 orders of magnitude. The speed has increased from 34 miles per hour ( $\mathrm{mph}$ ) on the Wright Brothers' airplane to $607 \mathrm{mph}$ on a $707-320 \mathrm{~B}$ in the $1960 \mathrm{~s}$, $1545 \mathrm{mph}$ on a Concorde in 1973, and 15,000 mph on a space shuttle in 198I. The improvements are I8X, 45X, and 44IX, respectively. The largest improvement is less than 3 orders of magnitude.

The parallelism between these two technologies is striking. They both evolved very rapidly, because of the tremendous commercial potential. In the Wright Brothers' days, when airplanes were made of cloth attached to frames, they were fragile and easily damaged, endangering the pilot, but they could be made inexpensively, requiring a minimal amount of sophistication. Similar are proximity-printing tools. The mask is easily contaminated or damaged by frequent accidental contacts to the wafer. However, proximityprinting tools are easy to build. As a result they are inexpensive.

Cloth-on-frame quickly became unitized-body; biplane, monoplane, pusher airplane, tractor airplane. The result of this evolution is well represented by the single-engine fighter plane of World War II, the Mustang. A P5ID can reach $437 \mathrm{mph}$ and has a range of 1300 miles. The pilot is safe from wind and debris, except for enemy fire. On this side, microlithography has switched from the defect-prone proximity printing to projection printing using full-wafer scanning. Even though the mask can still be contaminated accidentally, the frequency is inherently lower. The exposure field can be as large as 5 inches in width.

The ultimate in commercializing aviation with propeller engines can be represented by the Lockheed Constellation. This four-engine airliner is called one of the most beautiful aircraft ever built. It has a cruising speed of $314 \mathrm{mph}$ and a top speed of $366 \mathrm{mph}$ carrying over 100,000 lb gross weight for more than 3000 miles. The speed is not as high as the best single-engine fighters even though it can outrun many of them. For this aircraft, speed is not absolutely important. It carries a much larger payload and a longer range. For microlithography, the full-wafer scanner is replaced by stepand-repeat machines using a computer-controlled interferometric stage. The image is repeated many times on the wafer, more than the number of engines repeated on the Constellation. A pellicle protects the mask from particulate contaminations. Similarly, the passenger cabin of the Constellation is very safe and comfortable.

The speed of airplanes reached a plateau until the arrival of the jet age.The first jet-propelled commercial airplane in the United States was the Boeing 707. The latest model 707$320 \mathrm{~B}$ has a cruising speed of $607 \mathrm{mph}$, higher than almost all modern jet airliners except for the supersonic transport. For lithography, the compromise of numerical aperture and field size reached a limit. Step-and-scan systems took over. The field size increased to $26 \times 33 \mathrm{~mm}^{2}$ and the numerical aperture (NA) exceeded the previous limit to eventually reach the 0.93 regime. Keeping the same $\sin \theta$ at 0.93 , NA becomes 1.35 with water immersion. It can be further increased to beyond I.5 NA if high-index fluids and lens materials are successfully developed.

With jet propulsion the speed of sound is no longer a barrier. Military aircraft have been able to fly at better than Mach 2 during combat. The supersonic transport can cruise at Mach 2.2. However, the major commercial fleets all fly at subsonic speed. They do not even use the $607-\mathrm{mph}$ Mach 0.92 cruising speed of the 707-320B. Most of them, including the new 707-8 and 787, all fly at about Mach 0.84 or $555 \mathrm{mph}$. There are sound economic reasons not to blindly increase the speed of commercial airliners. However, the technology has not been standing still. Fuel economy, pollution, noise, passenger safety, comfort, and entertainment have all been improved. So, the present configuration is going to remain for a long time for economic reasons just as the Boeing 747 and 787 . What is the supersonic equivalent of a scanner? It is the use of double patterning to break through the resolution barrier, trading off throughput thus economy.

Double patterning is not a sustainable solution just as the supersonic transport. Then, what will take over as a longterm solution? Many have thought that it will be EUV.A fter all, the wavelength is dropped to an order of magnitude shorter than the water-immersion ArF wavelength. Many of the imaging tricks presumably can start over, pointing to more improvements. In aviation, one looks to rocketpowered aircrafts to reach higher speeds. A reusable, presumably economical crew-carrying aircraft is the space shuttle. After all, it can easily reach $15,000 \mathrm{mph}$, an order of magnitude higher than that of even the supersonic transport. After the maiden flight in 198I, the space shuttle has never reached mass-production status. This spans a quarter of a century. Since the first demonstration of EUV lithography in 1989, no less development has been devoted to EUV though not yet quite enduring a quarter of a century. Two $\alpha$ 
tools have been delivered. I would name them Columbia and Challenger. As to which is Columbia, history will tell.

I cannot find an aviation-equivalent of multiple e-beam direct write. Maybe it will not be a flying machine but just a hardworking one. After all, how can one fly at 10-30 wph? Flying or not is not an issue. Getting from one place to another safely with the minimum amount of time and cost is what counts.

Happy reading!

Burn J. Lin, Editor-in-Chief

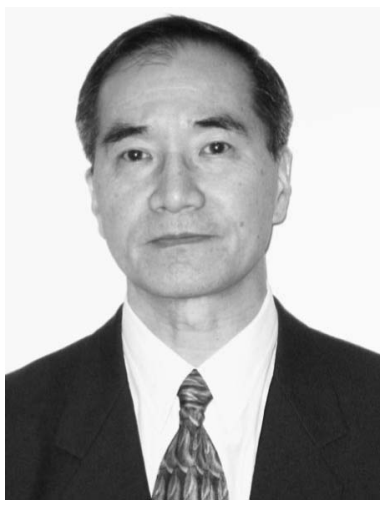

